non-groupable $7.9 \%$ and non-capsulated $44.7 \%$. Clonal complex was determined for 58 isolates. Independent predictor of $\mathrm{Nm}$ carriage: attendance at night clubs (adjusted OR: 3.38 , CI95\% $=1.28-8.93 ; P=0.013$ ); passive smoking at home (adjusted OR: 0.55 , CI $95 \%=0.32-0.93 ; P=0.025$ ). Preliminary data show a total of 10 different clonal complexes present among all serogroups in the 1-9 years group aged and 13 in 10-17 years.

Conclusion. Overall carriage was higher in the 10-17 years population. The non-encapsulated $\mathrm{Nm}$ was prevalent in both groups and serogroup B was the most frequent among the encapsulated. The results demonstrated a high diversity of N. meningitis in pharyngeal carriage.

Disclosures. All authors: No reported disclosures.

\section{The Frequency of Multifocal Disease and Pyogenic Hip Arthritis in}

\section{Neonates With Osteomyelitis}

Jiwoong Shin, $\mathrm{MD}^{1}$; Oded Scheuerman, $\mathrm{MD}^{2}$; Itzhak Levy, $\mathrm{MD}^{3}$ and Lorry Rubin,

MD, FIDSA ${ }^{4} ;{ }^{1}$ Pediatrics, Northwell Health - Cohen Children's, Queens, New York, ${ }^{2}$ Pediatrics B, Schneider Children's Medical Center of Israel, Petach Tikva, Israel, ${ }^{3}$ Schneider Children's Medical Center, Sackler Faculty of Medicine, Tel Aviv University, Petah Tikva, Israel, ${ }^{4}$ Cohen Children's Medicine Center of New York, Northwell Health, New Hyde Park, New York

Session: 247. Pediatric Bacterial Infections

Saturday, October 6, 2018: 12:30 PM

Background. Osteomyelitis and septic arthritis are important infections in neonates in intensive care units. The literature on neonatal osteoarticular infections during the past twenty years, a period during which there was an emergence of community-associated methicillin-resistant Staphylococcus aureus (MRSA), is limited. The purpose of this study was to describe a case series of neonatal osteoarticular infections during recent decades. In particular, we sought to describe the current microbiology, prevalence of multi-bone and contiguous joint involvement, and proportion of cases with pyogenic arthritis of the hip.

Methods. Multi-center retrospective chart review. Cases were identified through NICU registries at 2 tertiary/quaternary children hospitals between 1993 and 2017. The diagnosis required suggestive clinical findings plus radiological findings or positive blood, joint fluid, or bone culture.

Results. Thirty cases were identified. The median gestational age was 28 weeks, with $24(80 \%)$ cases occurring in premature babies. The median age at time of diagnosis was 33.5 days (range, 11-175) days.

The most common localized clinical findings were erythema (66\%) and swelling (60\%); the most common systemic finding was apnea (53\%). Seventy percent had osteomyelitis, $27 \%$ had both bone and joint involvement, and one had septic arthritis only. Eight babies (27\%) had an infection in more than one noncontiguous site. The most commonly identified bone was the femur (41\%) and joint was the knee $(20 \%)$ followed by hip (17\%). Of the 5 babies with hip infection, two were recognized more than 48 hours after initial presentation. An ultrasound of the hip was performed on 12 babies (40\%), including 4 out of the 5 who had surgical drainage of the hip.

Cultures of blood, joint fluid, and bone were positive in 23, 6, and 2 babies, respectively. Methicillin-susceptible S. aureus $(71 \%)$ was the most common pathogen followed by MRSA (21\%).

Conclusion. Neonates in a NICU with osteoarticular infection frequently have multiple sites of involvement. The hip joint is infected in a sizeable minority of babies with osteoarticular infection. In view of the importance of early diagnosis and surgical drainage of pyogenic hip arthritis, ultrasound of the hips should be considered in neonates in a NICU with osteoarticular infection at any body site.

Disclosures. All authors: No reported disclosures.

2317. Multicenter Retrospective Cohort Study of Pediatric Osteomyelitis Charles Treinen, $\mathrm{BSc}^{1}$; William Otto, $\mathrm{MD}^{2}$; Kari Simonsen, MD, FIDSA, FPIDS ${ }^{3}$; Nicole Le Saux, $\mathrm{MD}^{4}$; Jennifer Bowes, $\mathrm{M} \mathrm{Sc}^{5}$; Julianne Green, MD, $\mathrm{PhD}^{6}$; Mary Anne Jackson, MD, FIDSA, FPIDS ${ }^{2}$; Charles Woods, MD, MS, FIDSA, FSHEA, FPIDS $^{6}$; Jessica Snowden, $\mathrm{MD}^{7}$; Elizabeth Lyden, $\mathrm{MS}^{8}$ and H. Dele Davies, MD, MS, FIDS, FPIDS ${ }^{1} ;{ }^{1}$ Pediatrics, University of Nebraska Medical Center, Omaha, Nebraska, ${ }^{2}$ Pediatrics, Children's Mercy Hospital, Kansas City, Missouri, ${ }^{3}$ Pediatric Infectious Diseases, University of Nebraska Medical Center, Omaha, Nebraska, ${ }^{4}$ Infection Prevention and Control, Children's Hospital of Eastern Ontario, Ottawa, ON, Canada, ${ }^{5}$ Infectious Diseases, Children's Hospital of Eastern Ontario, Ottawa, ON, Canada, ${ }^{6}$ Pediatrics, University of Louisville School of Medicine, Louisville, Kentucky, ${ }^{7}$ University of Nebraska Medical Center, Omaha, Nebraska, ${ }^{8}$ Epidemiology, University of Nebraska Medical Center, Omaha, Nebraska

Session: 247. Pediatric Bacterial Infections Saturday, October 6, 2018: 12:30 PM

Background. There is controversy about the appropriate management of acute osteomyelitis in children.

Methods. Retrospective cohort study of presentation, management and outcomes of all patients admitted with acute osteomyelitis ( $<2$ weeks duration) during 2010 2016 at 4 US and Canadian tertiary care hospitals (hosp). Long-term complications (LTC) were defined as amputation, limp, chronic or secondary infection, or readmission. Overall complications included LTC, admission to ICU and delayed surgery ( $>72$ hours).

Results. 712 patients were admitted, with a median age of 8.0 years. There were significant differences in rates of initial use of MRI for diagnosis, MRSA, PICC insertion, hosp stay and IV antibiotic duration (Table 1). Clindamycin (45.7\%), cefazolin $(24.1 \%)$ and vancomycin $(13.7 \%)$ were the most common IV antibiotics used while clindamycin $(47.1 \%)$ and cephalexin $(38.6 \%)$ predominated for oral. The median age of patients with MRSA was similar to those without MRSA ( 8.2 vs. 7.8 years, $P=0.18$ ), but MSSA patients were older $(9.6$ vs. 6.9 years, $P<0.0001)$. Contiguous septic arthritis was more common in younger children $(6.8$ vs. 8.5 years, $P<0.001)$. MRSA patients had higher overall complication rates $(25.2 \%$ vs. $10.0 \%, P<0.0001)$, but long-term complications were unrelated to duration of IV or total antibiotics.

Table 1: Summary Results of Multi-center Study of Pediatric Osteomyelitis

\begin{tabular}{|c|c|c|c|c|c|}
\hline & $\begin{array}{l}\text { Children's } \\
\text { Hosp. of } \\
\text { Omaha }\end{array}$ & $\begin{array}{l}\text { Children's } \\
\text { Mercy, } \\
\text { Kansas City }\end{array}$ & $\begin{array}{l}\text { Norton } \\
\text { Children's }\end{array}$ & $\begin{array}{l}\text { Children's } \\
\text { Hosp. of } \\
\text { Eastern } \\
\text { Ontario }\end{array}$ & $P$-value \\
\hline $\begin{array}{l}\text { No. of patients } \\
\text { enrolled }\end{array}$ & 137 & 398 & 127 & 46 & - \\
\hline M: F ratio (\%) & $58.7: 41.2$ & $64.8: 35.1$ & $51.5: 48.4$ & $69.5: 30.4$ & 0.02 \\
\hline $\begin{array}{l}\text { Median age } \\
\text { (years) }\end{array}$ & 7.6 & 7.6 & 8.2 & 8.6 & 0.32 \\
\hline $\begin{array}{l}\% \text { of all initial } \\
\text { imaging MRI }\end{array}$ & $38.1 \%$ & $89.8 \%$ & $45.9 \%$ & $20.4 \%$ & $<0.0001$ \\
\hline $\begin{array}{l}\% \text { Abnormal, all } \\
\text { initial imaging }\end{array}$ & 75.8 & 87.4 & 80.0 & 68.8 & $<0.001$ \\
\hline MSSA rates (\%) & 40.3 & 46.0 & 45.0 & 50.0 & $<0.19$ \\
\hline MRSA rates (\%) & 10.7 & 17.1 & 23.0 & 0 & $<0.0001$ \\
\hline $\begin{array}{l}\text { Median hospital } \\
\text { stay (days) }\end{array}$ & 4.0 & 4.0 & 4.0 & 6.0 & $<0.0001$ \\
\hline $\begin{array}{l}\text { Median days of } \\
\text { IV antibiotics }\end{array}$ & 18.0 & 4.0 & 3.0 & 9.5 & $<0.0001$ \\
\hline $\begin{array}{l}\text { Median days } \\
\text { of total } \\
\text { antibiotics }\end{array}$ & 42.0 & 34.0 & 33.0 & 41.5 & $<0.0001$ \\
\hline $\begin{array}{l}\text { Frequency of } \\
\text { PICC insertion }\end{array}$ & $67.7 \%$ & $8.5 \%$ & $8.7 \%$ & $45.8 \%$ & $<0.0001$ \\
\hline $\begin{array}{l}\text { Long-term } \\
\text { complications }\end{array}$ & $6.1 \%$ & $4.3 \%$ & $7.1 \%$ & $10.5 \%$ & 0.18 \\
\hline
\end{tabular}

Conclusion. Despite significant variation in management, long-term complication rates were similar across US and Canadian sites with different MRSA rates. These data support equivalence of shorter ( $\leq 4$ days) duration of IV antibiotics and reduced need for PICC insertion for pediatric osteomyelitis.

Disclosures. All authors: No reported disclosures.

2318. Comparison of Musculoskeletal Infections Due to Non-Typhoidal Salmonella Species and Staphylococcus aureus in Immunocompetent Children Juri Boguniewicz, MD; Andrea Rubiano-Landinez, MD; Gabriella Lamb, MD and Sheldon L. Kaplan, MD, FIDSA; Baylor College of Medicine and Texas Children's Hospital, Houston, Texas

Session: 247. Pediatric Bacterial Infections

Saturday, October 6, 2018: 12:30 PM

Background. Non-typhoidal Salmonella species (NTS) rarely cause musculoskeletal (MSK) infections in healthy children. Data on clinical presentation and out comes of NTS MSK infections is limited to case reports and case series. No previous studies have directly compared children with NTS MSK infections to children with MSK infections due to Staphylococcus aureus (SA), the most common cause of MSK infections in children.

Methods. In a retrospective case-control study children aged 1 month to 18 years seen at Texas Children's Hospital from 2010 to 2017 with NTS MSK infections were compared with patients with SA MSK infections. Date of infection matched controls were selected 3:1. Patients with known hemoglobinopathies, immunodeficiencies or infections due to penetrating trauma or related to prosthetic devices were excluded. Logistic regression was used to evaluate associations between historical, clinical and laboratory variables and NTS or SA MSK infection.

Results. From 2010 to 2017, 27 cases of NST MSK infections were identified, 12 of which occurred in healthy children. The control group had 37 patients. The case and control groups had similar baseline demographics. Predictors of NTS MSK infection included exposure to reptiles (odds ratio [OR], 6.86; 95\% confidence interval $[\mathrm{CI}], 1.03-45.60)$ and a history of preceding diarrhea (OR, 7.25; 95\% CI, 1.12-47). No presenting signs or laboratory markers were identified as predictors of NTS MSK infection. Blood cultures were positive in $8(66.7 \%)$ of the NTS MSK cases. Length of hospital stay, duration of fever or complications did not differ significantly between the two groups and children with NTS MSK infections had a low rate of complications (16.7\%). Six (50\%) patients with NTS infections had unremarkable evaluations for hemoglobinopathies and immunodeficiencies.

Conclusion. Healthy children with NTS MSK infections often report a history of reptile exposure and preceding diarrhea compared with children with MSK infections due to SA. If such history is obtained, addition of a third-generation cephalosporin to empirically cover for NST should be considered pending blood and tissue cultures. In contrast to previous case reports and case series, children with NTS MSK infections had a lower rate of complications.

Disclosures. All authors: No reported disclosures. 